\title{
Pertechnegas lung clearance in different forms of interstitial lung disease
}

\author{
M.J. Thomeer*, B. Dehaes", L. Mortelmans", M. Demedts*
}

\begin{abstract}
Pertechnegas lung clearance in different forms of interstitial lung disease. M.J. Thomeer, B. Dehaes, L. Mortelmans, M. Demedts. C) ERS Journals Ltd 2002.

ABSTRACT: Interstitial lung diseases (ILD) are characterized by an acute or chronic inflammation of the alveolar capillary membrane, which affects the permeability of this membrane. A possible way to measure the permeability of the membrane is by radionuclide aerosol imaging. Pertechnegas, a gas composed of technetium-labelled carbon particles, has recently been proposed as a new ventilation agent to measure this lung clearance.

The clearance by pertechnegas in the four most common forms of ILD (eight patients with connective tissue disease, 10 with hypersensitivity pneumonitis, nine with idiopathic interstitial pneumonia and $\mathbf{1 0}$ with sarcoidosis) was measured and compared with $\mathbf{1 0}$ nonactive smoking controls. Because forced vital capacity (FVC), total lung capacity (TLC) and carbon monoxide diffusing capacity of the lung (DL,CO) are used in the assessment of functional severity of the ILD, the pertechnegas clearance was correlated with these lung-function indices.

It was found that the time to half clearance of pertechnegas of the lung is significantly decreased in idiopathic interstitial pneumonia $(p<0.0001)$, hypersensitivity pneumonitis $(p=0.0005)$ and connective tissue disease $(p=0.002)$ but not in sarcoidosis when compared with 10 nonsmoking controls. A significant correlation is also found between time to half clearance and FVC $(r=0.76 ; p<0.0001)$, TLC $(r=0.63 ; p<0.0001)$ and $D L, C O(r=0.75$; $p<0.0001$ ) for all groups together. For all subjects as a group, the time to half clearance is shorter in the upper lung zones than in the lower zones $(p<0.0001)$ and the ratio between both zones is not significantly different between the different types of disease.

These results indicate that pertechnegas clearance is increased in idiopathic interstitial pneumonia, hypersensitivity pneumonitis and connective tissue disease, but not in sarcoidosis and is related to the functional severity of the disease.

Eur Respir J 2002; 19: 31-36.
\end{abstract} \author{
Leuven, Leuven, Belgium. \\ Correspondence: M. Demedts \\ UZ Leuven \\ Afdeling Longziekten \\ Herestraat 49 \\ B-3000 Leuven \\ Belgium \\ Fax: 3216346803
}

Depts of *Respiratory and ${ }^{*}$ Nuclear Medicine, Universitaire Ziekenhuizen

Keywords: Collagen vascular disease hypersensitivity pneumonitis idiopathic pulmonary fibrosis interstitial lung disease

lung clearance pertechnegas

\section{Received: May 62001}

Accepted after revision September 19 2001
Many chronic forms of interstitial lung disease (ILD) may progress in an insidious way. Significant toxicity is associated with current therapy, such as corticosteroids and immunosuppressive/cytotoxic agents. In some forms of ILD (e.g. idiopathic pulmonary fibrosis (IPF)) the efficacy of these therapies is difficult to determine. Guiding therapy on disease activity in ILD is important but difficult, since no good disease-activity parameter exists in most of the ILD. Conversely, different studies have concluded that pulmonary function is correlated with survival: a lower carbon monoxide diffusing capacity of the lung ( $D \mathrm{~L}, \mathrm{CO})$, total lung capacity (TLC) and/or forced vital capacity (FVC) is associated with a worse prognosis [1-3].

The ILD are characterized by an acute or chronic inflammation of the interstitium, also called the alveolar capillary membrane [4]. A possible way to measure the alveolar capillary membrane permeability is by radionuclide aerosol lung imaging. The rate of the clearance of the aerosol is inversely related to the integrity of the alveolar capillary barrier [5-7]. In smokers and in patients with fibrotic lung disease this integrity is disrupted causing an increased clearance of the radionuclide aerosol $[8,9]$. Most studies use ${ }^{99 \mathrm{~m}}$ Tc-diethylenetriamine pentacetate (DTPA) [10], however, recently pertechnegas has been proposed as a promising agent to measure the lung clearance. Pertechnegas is produced by heating a saline solution of ${ }^{99 \mathrm{~m}} \mathrm{Tc}$-pertechnetate to $2500^{\circ} \mathrm{C}$ in a graphite crucible in a mixture of $4 \%$ oxygen in argon. The resulting radio-aerosol distributes in the lungs, crosses the alveolar capillary membrane and appears in the circulation [11-14]. Lung clearance by pertechnegas has only been assessed in ILD as a whole group [4] and it was found that patients with ILD had a significantly shorter time to half clearance versus controls $(5.8 \pm 2.2$ versus $8.5 \pm 2.4 \mathrm{~min})$. Yet the clearance rate has not been compared between welldescribed disease entities of ILD and has not been correlated with the severity of the disease measured by lung-function indices.

Therefore, the first objective in this study was to compare the time to half clearances measured by pertechnegas in the four most common forms of ILD (connective tissue disease (CTD), hypersensitivity 
pneumonitis (HP), idiopathic interstitial pneumonia (IIP) and sarcoidosis (SARC)) with nonsmoking controls. As a second study objective pertechnegas clearance was correlated with FVC, TLC and DL,CO because these lung-function tests are used as markers for the functional severity of the disease. As a third objective the time to half clearance in the upper lung zones was compared to that of the lower lung zones for each disease group. Indeed the topographic distribution of the lung clearance may be different for the different diseases.

\section{Methods}

\section{Subjects}

The protocol was reviewed and approved by the institutional review board for human studies. The data were collected prospectively. Subjects were aged between 18-85 yrs (pregnant females did not take part in the study) and were nonsmokers or former smokers (stopped smoking at least 6 months before the study). Only noncurrent smokers were included because active smoking increases the lung clearance [8].

Control subjects included: no currently active smokers, with no history of active or chronic pulmonary disease, and normal lung volumes (FVC, forced expiratory volume in one second (FEV1), TLC) and ( $D$ L,CO). Because the majority of patients with ILD are aged $\geqslant 50$ yrs most of the control subjects were also of a similar age. All patients with CTD have a restrictive lung-function defect (defined as a TLC $<$ $80 \%$ ) or a $D \mathrm{~L}, \mathrm{CO}<75 \%$ and/or the typical appearance of fibrosis and ground glass pattern on high-resolution computed tomography (HRCT). The diagnosis of lupus erythematosus disseminatus [15], systemic sclerosis [16] and Sjögren syndrome [17] is according to the criteria of the American Rheumatism Association. The criteria from BoHAN et al. [18] are used for the diagnosis of dermato-polymyositis.

The diagnosis of HP is based on the presence of ILD with a suggestive history and presentation [19], including: exposure to an agent that can induce HP, shortness of breath, the presence of precipitating antibodies and the presence of a high lymphocytosis in the bronchoalveolar lavage fluid (BALF), a restrictive lung function pattern, the presence of ground glass and/or fibrosis pattern on HRCT. If the clinical, BALF and/or radiological features were not suggestive, the diagnosis of HP was based on lung biopsy [20] or a specific inhalation challenge test.

The diagnosis of IIP was based on the following criteria: nonspecific interstitial pneumonia and desquamative interstitial pneumonia proven by lung biopsy and conforming to the histological criteria described by KATZENSTEIN et al. [21]. The diagnosis of usual interstitial pneumonia was based on the criteria of the American Thoracic Society/European Respiratory Society (ATS/ERS) consensus statement [22].

The diagnosis of SARC was based on a compatible clinical presentation of multi-organ involvement, histological demonstration of noncaseating granulomas, and exclusion of other diseases capable of producing a similar histological or clinical picture. If clinical, BALF and/or radiological features alone were diagnostic for SARC, no biopsy was performed [23]. All radiological stages, including stage I, were accepted in the study.

\section{Pertechnegas}

Pertechnegas is produced from a generator manufactured and supplied by Tetley Technologies (Sydney, Australia). Sodium ${ }^{99 \mathrm{~m}}$ pertechnetate $(0.1 \mathrm{~mL}, 400-$ 500 megabecquerel (MBq)) is added to a graphite crucible after it is rinsed with ethyl alcohol. Preheating it for 6 min evaporates the liquid component and an aerosol of technetium-labelled carbon particles is produced by heating to $2,500^{\circ} \mathrm{C}$ in an atmosphere of $3-5 \%$ oxygen in argon [11].

The subject inhaled pertechnegas for 2-4 breaths from residual volume (RV) to TLC in supine position via a mouthpiece (with an one-way valve system and a noseclip) that was connected to the pertechnegas generator. This delivered $\sim 40-50 \mathrm{MBq}$ activity to the lungs reaching a count rate of generally $>2,000$ counts $\cdot \mathrm{s}^{-1}$. Posterior images were obtained with a gamma camera with the patient in supine position at a frame rate of $1 \mathrm{~s} \cdot$ frame $^{-1}$ for $5 \mathrm{~min}$ followed by $60 \mathrm{~s} \cdot$ frame $^{-1}$ for $40 \mathrm{~min}$. Images were acquired with a $64 \times 64$ matrix. Regions of interests were drawn around each lung and around the left and right upper and lower lung zones separately. A time-activity curve without background correction for both lungs was generated [9]. Background was measured over the liver and increased steadily to a value of only $3.47 \pm 1.48 \%$ (mean \pm SD) of the remaining lung activity after $45 \mathrm{~min}$. Since this value was so low and background correction did not influence the clearance, data without correction are shown in this study. A line of best mono-exponential fit was derived from all points from the peak initial activity to the last measured activity at $45 \mathrm{~min}$ by applying a nonlinear regression model of one-phase exponential decay using the following equation:

$$
\mathrm{A}_{\mathrm{t}}=\mathrm{A}_{0} \mathrm{e}^{-\mathrm{kt}} \text { and } \mathrm{t}_{1 / 2}=0.693 \mathrm{k}
$$

where $A_{t}$ is the tracer activity at time $t$ and $A_{0}$ the activity at time 0 . The time to half-clearance $\left(t_{1 / 2}\right)$ is calculated from each exponential fit and converted to per cent clearance $\cdot \mathrm{min}^{-1}$ by using the formula:

$$
\text { Clearance }=100\left(1-\mathrm{e}^{-0.693 / t_{1 / 2}}\right)
$$

A typical example is shown in figure 1.

\section{Respiratory function tests}

In all subjects respiratory function tests (FVC, FEV1, TLC and DL,CO) were performed within 2 weeks of the pertechnegas measurement. These data are expressed as percentages of predicted values adopted by the ERS [24]. 


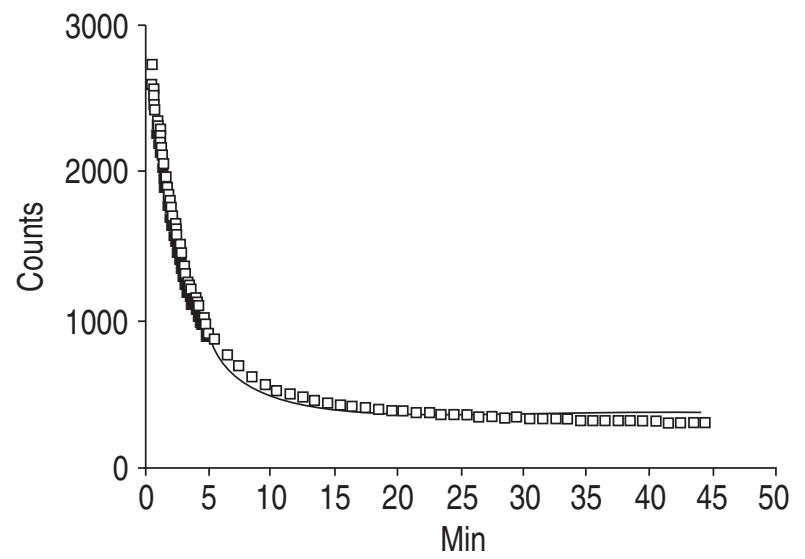

Fig. 1.- Time activity curve of the whole lung from a patient suffering from progressive systemic sclerosis. From this curve a line of best exponential fit is derived from peak initial activity and the last measured activity at $45 \mathrm{~min}$. A time to half clearance $\left(\mathrm{t}_{1 / 2}\right)$ is calculated and converted to $\%$ clearance $\min ^{-1}$ using the formula: Clearance $=100\left(1-\mathrm{e}^{-0.693 / \mathrm{t} / \mathrm{s}}\right) \cdot \mathrm{t}_{1 / 2}: 2.17 \mathrm{~min} ; 95 \%$ confidence interval: $2.13-2.21$; clearance: $27.3 \% \cdot \mathrm{min}^{-1} ; \mathrm{R}^{2}=0.99$.

\section{Statistics}

Numbers are expressed in medians and ranges (minimum-maximum). The mean times to $t_{1 / 2}$ between groups were analysed using the Mann-Whitney U-test.

To correlate the different lung function indices with the time to $t_{1 / 2}$ for pertechnegas the Pearson correlation coefficient was calculated after the data were checked by the Kolmogorov-Smirnov test for Gaussian distribution.

\section{Results}

The inhalation of pertechnegas to all of the 47 subjects was uncomplicated and did not provoke any breathing difficulties. Most of the subjects (35 of 47) could reach $>2,000$ counts of pertechnegas over the lungs after three breathing manoeuvres (mean peak count \pm SD: $3,699 \pm 2,470$ counts). A time/activity curve could be generated in every patient and fitted monoexponentially (mean $\pm \mathrm{SD} ; \mathrm{R}^{2}=0.98 \pm 0.03$ ).

The subject characteristics are presented in table 1 . None of the subjects were current smokers. The eight patients with CTD consisted of four with systemic

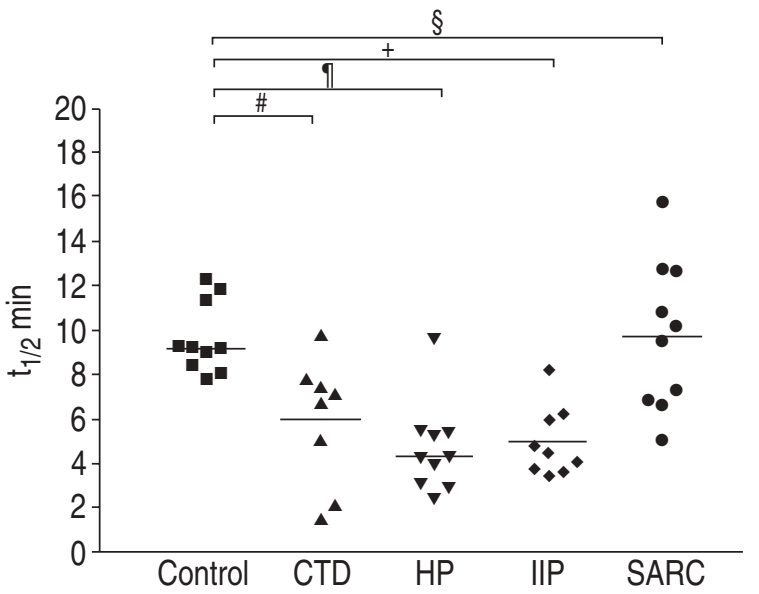

Fig. 2. - Time to half clearance $\left(t_{1 / 2}\right)$ measured for each group, with the mean numbers expressed by the horizontal lines. CTD: connective tissue disease, HP: hypersensitivity pneumonitis; IIP: idiopathic interstitial pneumonia, SARC: sarcoidosis. ${ }^{\#}$ : $\mathrm{p}=0.003$; ॠ: $\mathrm{p}=0.0005 ;{ }^{+}: \mathrm{p}<0.0001 ;{ }^{\S}: \mathrm{p}=0.97$.

lupus erythematosus, two with progressive systemic sclerosis, one with polymyositis-dermatomyositis and one with Sjögren's disease. Most of the 10 patients with HP were pigeon breeders. The nine patients with IIP consisted of five with usual interstitial pneumonia, three with nonspecific interstitial pneumonia and 1 with a desquamative interstitial pneumonia. The 10 patients with SARC consisted of four with chest radiographic stage I, five with stage II and one with stage III [23].

The individual lung clearances per group are shown in figure 2 . The values for $t_{1 / 2}$ were controls: 9.2 (7.8-12.3) min; CTD: 6.9 (1.5-9.8) min; HP: 4.3 (2.5-9.7) min; IIP: 4.5 (3.5-5.0) min; and SARC: 9.9 (8.2-15.8) $\mathrm{min}$; (in stage I the values were 9.5 (5.1-12.7) $\mathrm{min}$ and in stage II-III 10.8 (6.9-15.8) min). The respective lung clearance in controls was $7.3(5.5-8.5) \% \cdot \mathrm{min}^{-1}, 9.6(6.8-37.5) \% \cdot \mathrm{min}^{-1}$ in CTD, $14.8(6.9-23.9) \% \cdot \mathrm{min}^{-1}$ in HP, $14.4(8.1-18.1) \% \cdot \mathrm{min}^{-1}$ in IIP and $6.8(4.3-12.8) \% \cdot \mathrm{min}^{-1}$ in SARC (in stage I the values were $7.0(5.3-12.8) \% \cdot \mathrm{min}^{-1}$ and in stage II-III $\left.6.2(4.3-9.6) \% \cdot \mathrm{min}^{-1}\right)$. Compared to nonsmoking controls the $t_{1 / 2}$ of the lung measured by pertechnegas was significantly decreased in CTD, HP, and IIP, but not in SARC.

Because $t_{1 / 2}$ was not significantly decreased in SARC, the Pearson correlation coefficient between

Table 1. - Characteristics of the study population

\begin{tabular}{lccccc}
\hline & Control & CTD & HP & IIP & Sarcoidosis \\
\hline Subjects (F) & $10(4)$ & $8(5)$ & $10(4)$ & $9(4)$ & $10(6)$ \\
Age yrs & $59(32-68)$ & $56(44-77)$ & $68(32-85)$ & $65(42-84)$ & $48(27-74)$ \\
FVC \% & $113(83-142)$ & $78(60-103)$ & $70(52-114)$ & $60(41-107)$ & $98(82-123)$ \\
FEV1\% & $98(62-150)$ & $74(36-97)$ & $67(40-101)$ & $61(41-104)$ & $91(69-117)$ \\
TLC \% & $100(70-115)$ & $81(64-108)$ & $67(54-118)$ & $60(48-105)$ & $91(71-114)$ \\
$D$ L,CO \% & $101(77-119)$ & $35(23-71)$ & $43(35-68)$ & $37(19-66)$ & $87(53-103)$ \\
\hline
\end{tabular}

Data are presented as median (range); lung function indices are expressed in per cent predicted; CTD: connective tissue disease; HP: hypersensitivity pneumonitis; IIP: idiopathic interstitial pneumonia; SARC: sarcoidosis; FVC: forced vital capacity; FEV1: forced expiratory volume in one second; TLC: total lung capacity; DL,CO: carbon monoxide diffusing capacity of the lung. 

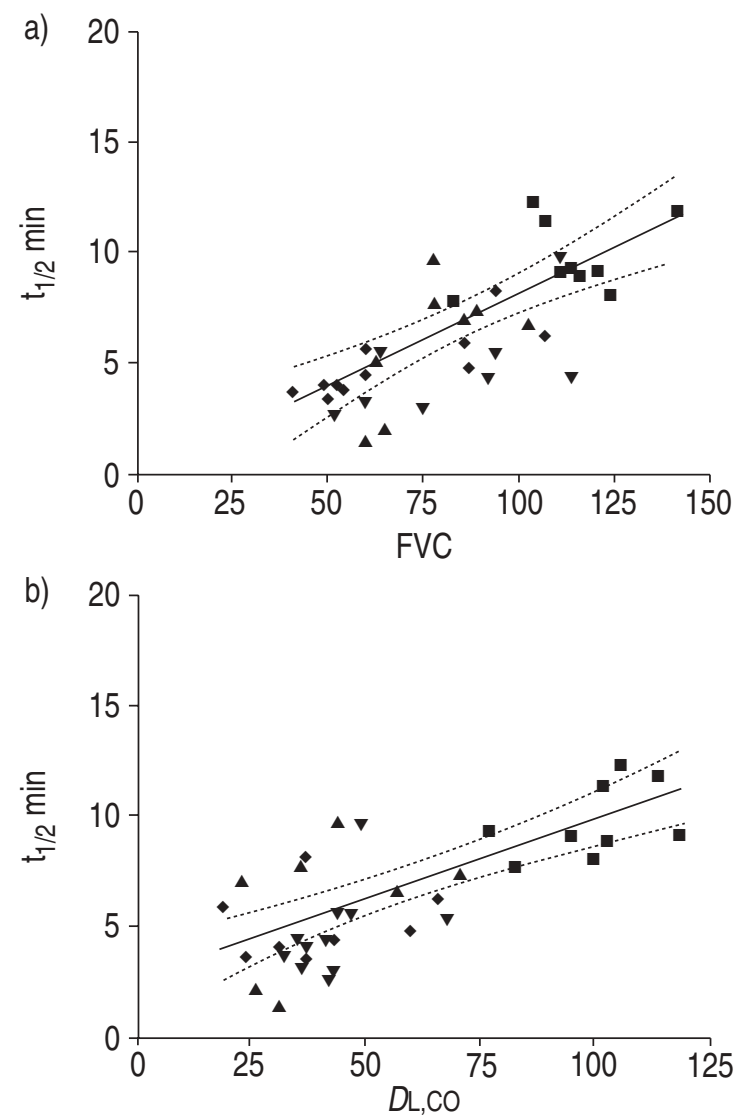

Fig. 3.-Correlation between time of half clearance $\left(t_{1 / 2}\right)$ and a) forced vital capacity (FVC) and b) carbon monoxide diffusing capacity of the lung ( $D \mathrm{~L}, \mathrm{CO})$ (expressed in \% predicted) expressed by the Pearson correlation coefficient $(\mathrm{r})$. $\boldsymbol{\Delta}$ : connective tissue disease; $\boldsymbol{\nabla}$ : hypersensitivity pneumonitis; $\bullet$ : idiopathic interstitial pneumonia; : control. $\mathrm{r}=0.76$; $>0.001$; D L,CO: Pearson correlation, $\mathrm{r}=0.75 ; \mathrm{p}<0.0001$.

$\mathrm{t}_{1 / 2}$ and FVC, TLC, DL,CO with and without SARC was calculated. Interestingly, the value for the different groups all falls along one regression slope. This is shown in figure 3 for the relationships of $t_{1 / 2}$ with FVC and DL,CO in all groups, except for SARC. All correlations were significant between $t_{1 / 2}$ and $\mathrm{FVC}$ $(\mathrm{r}=0.62 ; \mathrm{p}<0.0001), \quad$ TLC $\quad(\mathrm{r}=0.51 ; \mathrm{p}<0.0001)$ and $D$ L,CO $(\mathrm{r}=0.68 ; \mathrm{p}<0.0001)$ with SARC, between $\mathrm{t}_{1 / 2}$ and FVC $(\mathrm{r}=0.76 ; \mathrm{p}<0.0001)$, TLC $(\mathrm{r}=0.63 ; \mathrm{p}<0.0001)$ and DL,CO $(\mathrm{r}=0.75 ; \mathrm{p}<0.0001)$ without SARC. Figure 3 shows the correlation between FVC, TLC, $D$ L,CO and $t_{1 / 2}$ without SARC.

The ratio of the $t_{1 / 2}$ in the upper lung zones over those in the lower zones was control: $1.0(0.5-2.7)$; CTD: 1.4 (0.9-2.7); HP: 1.3 (0.7-2.6); IIP: 1.2 (0.8-6.6); and SARC: 1.0 (0.4-2.6). No significant difference between the different groups was seen. In all groups $(n=47)$ the $t_{1 / 2}$ was shorter in the lower lung zones than in the upper zones $(\mathrm{p}<0.0001)$.

\section{Discussion}

This study shows that the $t_{1 / 2}$ of the lung measured by pertechnegas is significantly decreased (i.e. faster lung clearance) in CTD, HP and IIP compared with nonsmoking controls (fig. 2). Mouratidis et al. [4] also found evidence that $t_{1 / 2}$ measured by pertechnegas was decreased in 22 patients with ILD. The present study compares, for the first time, the four most common forms of ILD. Interestingly no decrease in $t_{1 / 2}$ in patients with SARC was found (fig. 2). Another new finding in this study was that FVC $(r=0.76)$, TLC $(\mathrm{r}=0.63)$ and $D \mathrm{~L}, \mathrm{CO}(\mathrm{r}=0.75)$ were significantly correlated with the $t_{1 / 2}$ of the clearance indicating that all groups fall along the same regression line. A third new finding was that in all groups the ratio of $t_{1 / 2}$ of the upper zones over the lower zones was $\geqslant 1$.

A mean clearance rate of $7.09 \% \cdot \mathrm{min}^{-1}$ (or a median of $7.3 \% \cdot \mathrm{min}^{-1}$ ) was found in the nonsmoking control subjects. This finding was similar to the clearance rate of $9.2 \% \cdot \mathrm{min}^{-1}$ estimated by WIDDICOMBE [25]. This estimation is based on an assumed permeability coefficient for the alveoli of $-0.23 \times 10^{-7} \mathrm{~cm} \cdot \mathrm{s}^{-1}$ and a $0.15 \mu \mathrm{m}$ thick alveolar capillary layer obtained from physiological measurements [26]. WIDDICOMBE [25] stressed, that these estimates are nine times higher than the values obtained with DTPA $(0.59$ $1.56 \% \cdot \mathrm{min}^{-1}$ ) and suggested that this may be due to the fact that the majority of the particles are larger (mass median aerodynamic diameter (MMAD of $0.7 \mu \mathrm{m})$ than those of pertechnegas $(80 \%$ of particles have a MMAD $<0.1 \mu \mathrm{m})$, which means that the majority of the pertechnegas particles are deposited in the alveoli [12, 27]. The other advantages of pertechnegas over DTPA are that no aerosol device for administration of the tracer to the patient (with more risk of surrounding contamination) is needed and that the inhalation time is shorter. When using DTPA the inhalation time must be long enough to reach a reliable peak count over the lungs. These advantages of pertechnegas over DTPA apparently permit a marked improvement in image quality, tracer counting and an easier administration of the radio-aerosol [12].

Lung clearance of a radiotracer measures the alveolar capillary permeability, and its increase in ILD can be used as a marker of inflammation or fibrosis of the alveolar capillary barrier. Indirect arguments that lung clearance is correlated with inflammation are present: JoNEs et al. [28] showed, in animal studies, that the lung clearance was correlated with different concentrations of tracheal instilled hydrochloric acid and hypotonic saline, which were used to cause alveolar inflammation. Studies in humans with acute respiratory distress syndrome showed that the lung clearance correlated with the severity of the hypoxia and parenchymal lesions [6]. In patients with progressive systemic sclerosis, lung clearance was higher in fibrosing alveolitis than in pulmonary vascular disease alone, but both were increased compared to normal controls [7].

It was found that lung clearance measured by pertechnegas was significantly correlated with FVC, TLC and DL,CO and that functional impairment was associated with a poorer prognosis. Very interestingly all groups fall along the same regression line indicating that the relationship between lungfunction abnormalities and lung clearance are similar. 
Therefore clearance seems to be a good measure of functional severity.

An increase in mean clearance was not found in patients with SARC. The $t_{1 / 2}$ was characterized by a wide range of variation in contrast to the control subjects (figure 2). One possible explanation is that the alveolar capillary membrane permeability is not affected in the same way as in the other three forms of ILD in this study. SARC is generally characterized by the presence of tight, well-formed granulomas that are spread in the perilymphatic interstitium [23]. These granulomas probably do not or only partially affect the alveolar membrane in contrast to the diffuse process in the other forms of ILD in this study. Another more plausible explanation is that the patients with SARC did not have a clear functional impairment and thus had no marked interstitial inflammation. Indeed in the SARC patients the $t_{1 / 2}$ are also related to the lung-function impairment. This cross-sectional study without follow-up does not allow for the identification of patients who are likely to respond or deteriorate. The only indirect argument that can be made is that since worse lung-function impairment is associated with poorer prognosis [1, 29], a faster pertechnegas clearance probably also indicates a poorer prognosis.

Interestingly, in all groups the $t_{1 / 2}$ was shorter in the lower lung zones than in the upper (apical) zones despite the fact that the radiographical, topographical distribution of the different disease entities may be different. The IIP generally affects the peripheral basal lung zones more while SARC has a perihilar distribution. In acute HP the radiographical infiltrates are mostly in the basal lung zones and shift towards the upper zones when the disease becomes more chronic. The apicobasal gradient in the $t_{1 / 2}$ can probably be explained by the apico-basal ventilationperfusion gradient. The relatively larger perfusion in the lower zones may explain the faster washout of pertechnegas in these zones.

This study also has some limitations. The data presented are from four different disease types, which are in a variety of disease stages: some of the patients are not treated, others have been treated for years. The present study could not conclude that the measured clearance is correlated with disease activity, since no validated score for activity for ILD exists and since a follow-up study was not performed.

To conclude, a decreased $\mathrm{t}_{1 / 2}$ (i.e. an increased lung clearance) in patients with CTD, HP and IIP was found. An increased lung clearance was not found in SARC, perhaps because in these patients the alveolar capillary membrane is not or only partially affected in contrast to the three other forms of ILD or because of a better functional status of these patients. The $t_{1 / 2}$ was shorter in all groups in the lower as compared to the upper lung zones and was probably related to the topographical ventilation-perfusion ratio. It was also found that the $t_{1 / 2}$ of pertechnegas was significantly correlated with FVC, TLC and DL,CO, which represented the functional severity of the disease.

Further studies are needed to assess pertechnegas lung clearance in relation to further evaluation of the disease and to a validated disease activity index for the different forms of interstitial lung diseases.

\section{References}

1. Erbes R, Schaberg T, Loddenkemper R. Lung function tests in patients with idiopathic pulmonary fibrosis. Chest 1997; 111: 51-57.

2. Schwartz DA, Helmers RA, Galvin J, et al. Determinants of survival in idiopathic pulmonary fibrosis. $\mathrm{Am}$ J Respir Crit Care Med 1994; 149: 450-454.

3. Thomeer M, Demedts M. Survival in interstitial lung diseases: correlation with findings at diagnosis. Sarcoidosis 1997; 14: S37.

4. Mouratidis B, Lising J, Nogrady S, Hurwitz M. Increased pertechnegas lung clearance in interstitial lung disease. Clin Nucl Med 1999; 24: 105-108.

5. Barrowcliffe MP, Jones JG. Solute permeability of the alveolar capillary barrier. Thorax 1987; 42: 1-10.

6. Barrowcliffe MP, Jones JG. Pulmonary clearance of ${ }^{99 \mathrm{~m}} \mathrm{Tc}$-DTPA in the diagnosis and evolution of increased permeability pulmonary oedema. Anaesth Intensive Care 1989; 17: 422-432.

7. Kon OM, Daniil Z, Black CM, du Bois RM. Clearance of inhaled technetium-99m-DTPA as a clinical index of pulmonary vascular disease in systemic sclerosis. Eur Respir J 1999; 13: 133-136.

8. Jones JG, Minty BD, Lawler P, Crawley JC, Hulands $\mathrm{G}$, Veall N. Increased alveolar epithelial permeability in cigarette smokers. Lancet 1980; 1: 66-68.

9. Kotzerke J, van den Hoff J, Burchert W, Wagner TOF, Emter M, Hundeshagen H. A compartmental model for alveolar clearance of pertechnegas. $\mathrm{J} \mathrm{Nucl}$ Med 1996; 37: 2066-2071.

10. Coates $\mathrm{G}, \mathrm{O}^{\prime}$ Brodovich H. Measurement of pulmonary epithelial permeability with ${ }^{99 \mathrm{~m}}$ Tc-DTPA aerosol. Semin Nucl Med 1986; 16: 275-284.

11. Mackey DWJ, Jackson P, Baker RJ, et al. Physical properties and use of pertechnegas as a ventilation agent. J Nucl Med 1997; 38: 163-167.

12. Monaghan P, Provan I, Murray C, et al. An improved radionuclide technique for the detection of altered pulmonary permeability. J Nucl Med 1991; 32: 19451949.

13. Scalzetti EM, Gagne GM. The transition from technegas to pertechnegas. $J$ Nucl Med 1995; 36: 267-269.

14. Fanti S, Conpagnone G, Pancaldi D, et al. Evaluation of lung clearance of inhaled pertechnegas. Ann Nucl Med 1996; 10: 147-151.

15. Tan EM, Cohen AS, Fries JF, et al. The 1982 revised criteria for the classification of systemic lupus erythematosus. Arthritis Rheum 1982; 25: 1271-1277.

16. Subcommittee for scleroderma criteria of the American Rheumatism Association Diagnostic and Therapeutic Criteria Committee. Preliminary criteria for the classification of systemic sclerosis (scleroderma). Arthritis Rheum 1980; 23: 581-590.

17. Fox RI, Robinson CA, Curd JG. Sjögren's syndrome. Proposed criteria for classification. Arthritis Rheum 1986; 29: 577-585.

18. Bohan A, Peter JB. Polymyositis and dermatomyositis. $N$ Engl J Med 1975; 292: 344-347.

19. Arnett FC, Edworthy SM, Bloch DA. The American Rheumatism Association 1987 revised criteria for the 
classification of rheumatoid arthritis. Arthritis Rheum 1988; 31: 315-324.

20. Coleman A, Colby TV. Histologic diagnosis of extrinsic allergic alveolitis. Am J Surg Pathol 1988; 12: $514-518$.

21. Katzenstein AA, Myers JL. Idiopathic pulmonary fibrosis: clinical relevance of pathologic classification. Am J Respir Crit Care Med 1998; 157: 13011315.

22. ATS/ERS Committee. Idiopathic pulmonary fibrosis: diagnosis and treatment. International consensus statement. Am J Respir Crit Care Med 2000; 161: 646-664.

23. ATS/ERS/WASOG Committee. Statement on sarcoidosis. Am J Respir Crit Care Med 1999; 160: 736-755.

24. Official Statement of the European Respiratory Society. Standardized lung function testing. Eur Respir J 1993; 6: 5-100.
25. Widdicombe J. Airway and alveolar permeability and surface liquid thickness: theory. J Appl Physiol 1997; 82: 3-12.

26. Stephens RH, Benjamin AR, Walters DV. Volume and protein concentration of epithelial lining liquid in perfused in situ postnatal sheep lungs. J Appl Physiol 1996; 80: 1911-1920.

27. Lloyd JJ, Shields RA, Taylor CJ, James JM, Testra HJ. Technegas and pertechnegas particle size distribution. Eur J Nucl Med 1995; 22: 473-476.

28. Jones JG, Berry M, Hulands GH, Crawley JC. The time course and degree of change in alveolar-capillary membrane permeability induced by aspiration of hydrochloric acid and hypotonic saline. Am Rev Respir Dis 1978; 118: 1007-1013.

29. Thomeer M, Vansteenkiste J, Demedts M. Actuarial survival of interstitial lung disease. Am J Respir Crit Care Med 1998; 157: A279. 